CRÍTICA, Revista Hispanoamericana de Filosofía

Vol. XXV, No. 74 (agosto 1993): 3-21

\title{
MÉTODO, EVOLUCIÓN Y PROGRESO EN LA CIENCIA (2a. Parte)*
}

SERGio MARTínEZ

Instituto de Investigaciones Filosóficas

7. Un caso de estudio: Bateson vs. Morgan acerca de las leyes de Mendel

Arriba he presentado una serie de ejemplos que muestran las dificultades que tiene que afrontar la idea de que la estabilidad de los fenómenos puede reducirse a la manipulación de leyes. Esto refuerza nuestra postura elaborada a partir de un tema central en la filosofía de la ciencia de L. Fleck en secciones anteriores (sobre todo en la sección cuatro): la idealización tradicional entre leyes y condiciones de aplicación no tiene fundamento en nuestra experiencia. Es necesario abandonar este supuesto para llegar a tener una idea menos distorsionada de lo que es buena parte de la ciencia. A continuación muestro la importancia de hacer esta distinción entre estabilidad de fenómenos y manipulación de leyes para la reconstrucción de un pasaje clave en la historia de la ciencia del siglo XX.

* Este trabajo es parte de una investigación apoyada por la DGAPA (UNAM) por medio de los proyectos IN600289 y IN600192. La primera parte de este artículo apareció en el número anterior de Crítica. 
Las leyes de Mendel constituyen el centro de atención de la ciencia en ciernes de la genética en la primera década de este siglo. Muy pronto se descubre que la segunda ley (que se formulaba diciendo que los caracteres hereditarios se distribuyen independientemente) no concuerda con los experimentos. Hay quienes toman esto como refutación de la segunda ley de Mendel. Sin embargo, muchos genetistas, convencidos de la importancia de las leyes de Mendel, tratan de modificarlas de manera tal que se adecuen a la evidencia experimental. Bateson, por ejemplo, trata de encontrar una generalización de la razón geométrica postulada por la ley. En lugar de la razón $9: 3: 3: 1$ que la ley postula para dihíbridos, Bateson propone varias complicadas razones geométricas que generalizarían la ley de tal manera que se adecuen a la evidencia experimental. Ninguna de estas propuestas es satisfactoria. ${ }^{l}$

La estrategia de Morgan y de sus colaboradores es diferente. Ellos tratan de explicar la supuesta ley como el efecto estable de un mecanismo muy complejo de transmisión de los caracteres hereditarios. Este mecanismo puede resumirse en lo que ellos llaman "el mapeo del ligamiento". Los resultados experimentales se explican, no por medio de una generalización de la ley, sino mediante una reinterpretación de la ley como una descripción de la interacción de diferentes mecanismos que actúan en el nivel cromosómico y que hacen más probables unas distribuciones que otras. Lo que Bateson veía como desviaciones de la ley, que requerían la formulación de una ley más general, Morgan lo vio como evidencia de la actuación de diferentes mecanismos (recombinación, no disyunción, etc.). Ahora sabemos

${ }^{1}$ Una discusión detallada de las propuestas de Bateson y otros, y de la estrategia de Morgan, se encuentra en el capítulo 9 de Darden, 1991. Una presentación de los aspectos cruciales de este tema con un énfasis diferente se encuentra en Carlson, 1966, y en cualquier libro de historia de la genética. 
que Morgan estaba básicamente en lo correcto. Lo que hizo Morgan fue proponer el grupo de mecanismos que, como se vio posteriormente, pudo utilizarse como punto de partida para el establecimiento de otros fenómenos que, a su vez, confirieron estabilidad y precisión a los mecanismos postulados inicialmente.

Llamaremos fenómeno fundador al resultado de un mecanismo postulado que lleva a la localización de un nivel de organización en el cual el mecanismo postulado inicialmente se estabiliza por medio del atrincheramiento del mecanismo en cuestión en otros mecanismos que, a su vez, desembocan en la producción de nuevos fenómenos, algunos de los cuales por lo menos pueden manipularse. ${ }^{2}$ El descubrimiento de los mecanismos de "recombinación", "no disyunción", etc., permitió refinar el mecanismo inicialmente postulado por la escuela de Morgan en el nivel cromosómico (sobre la base de trabajos de Boveri y Sutton). Este primer mapeo de ligamiento en el nivel cromosómico sería un ejemplo típico en nuestra terminología de un fenómeno fundador que no debería interpretarse como el descubrimiento de una ley.

Galileo creó un fenómeno fundador, la ley de la caída libre de los cuerpos, en el sentido de que propuso un tipo de transformaciones (procesos dinámicos) que pueden derivarse de un mecanismo básico: un plano inclinado. Galileo formula la ley de la caída libre de los cuerpos a partir de la descomposición de una serie de hechos y procesos (de caída) en una componente vertical en la que actúa la gravedad y una componente horizontal que es manipulable. Esto puede interpretarse en términos de la manipulación de una ley. Ésta es la manera como se interpreta en el contexto de

${ }^{2}$ Nótese que la designación de un fenómeno como "fenómeno fundador" es un recurso historiográfico, no la designación de un fenómeno en particular fuera de una reconstrucción de la historia de la ciencia. 
la tradición de la mecánica teórica. Pero no tiene por qué interpretarse así. El trabajo de Galileo puede interpretarse como si estableciera un fenómeno fundador. En el caso de Morgan, sin embargo, es más claro que no debe interpretarse el establecimiento del mecanismo de transmisión (en el nivel cromosómico) como la manipulación de una ley, sino como la consolidación (atrincheramiento) de fenómenos estables en un nivel cromosómico de organización. En este caso, interpretar el fenómeno del mapeo del ligamiento como la manipulación de una ley sería engañoso. Nos llevaría en la dirección en la que se perdieron Bateson y muchos otros teóricos que intentaban seguir las huellas de la física (y en particular la concepción tradicional de una explicación basada en leyes) demasiado cerca. El hecho es que el mapeo de ligamiento no describe una ley en el sentido tradicional en la física, sino más bien describe la evolución de restricciones (constraints) asociadas con la transmisión de caracteres.

La segunda ley de Mendel en la formulación de Morgan no es una idealización de cierto tipo de situaciones en las que cierto efecto se da con necesidad, sino más bien es la descripción de posibles maneras (cuantificables) en las que una serie de mecanismos pueden interferir mutuamente en la transmisión de los caracteres hereditarios.

El mecanismo puede interpretarse como la acción de una ley a la que no tenemos acceso sino mediante idealizaciones. Pero entonces se pierde de vista el contexto del mecanismo que debe verse como parte esencial del concepto de mecanismo. Pensar que el contexto del mecanismo, el nivel de la realidad en que actúa el mecanismo, es dispensable en una descripción de la realidad — porque la objetividad se reduce en última instancia a las leyes que no se subordinan a ningún contexto (y en este sentido son "universales") es simplemente repetir un dogma que hoy debemos reco- 
nocer como poco fructífero. Bateson no es el único que se ha perdido al tratar de reducir mecanismos a leyes. ${ }^{3}$

\section{El concepto de atrincheramiento}

Un fenómeno $A$ se atrinchera en otro fenómeno $B$ cuando $A$ entra en la creación de $B$. La creación de un fenómeno es un proceso histórico. La creación de una nueva variedad de planta requiere la presencia de mecanismos de variación y selección (y de otros mecanismos evolutivos). La creación del efecto fotoeléctrico es un largo proceso por medio del cual se va refinando el mecanismo de producción del fenómeno. Este proceso de refinamiento puede verse como un proceso adaptativo. A cada paso se exploran una serie de refinamientos posibles y los que son más exitosos, en términos de la producción de nuevos fenómenos, son los que tienden a atrincherarse. Los fenómenos construidos por los científicos constituyen una jerarquía diferente de las jerarquías biológicas, pero pueden verse como una generalización de estas jerarquías. ${ }^{4}$ La jerarquía taxonómica

${ }^{3}$ Galileo pensaba que la ley de la caída libre de los cuerpos actuaba en los cuerpos sobre la superficie de la tierra, pero que el movimiento de los planetas se explicaba por principios muy diferentes. Es por ello que si entendemos "la ley de la caída libre" como un fenómeno fundador, y no propiamente como una ley, no tiene sentido decir (como Feyerabend lo ha sostenido) que "la ley de la caída libre de los cuerpos", como cualquier otra ley con pretensión de universalidad, está subdeterminada por la evidencia. Un mecanismo no es simplemente falso o verdadero y por lo tanto el problema que plantea su postulación no puede entenderse en términos de la subdeterminación de la evidencia. Un fenómeno fundador se atrinchera en la historia de la ciencia y por lo tanto se vuelve un mecanismo adecuado para la descripción de ciertos procesos en cierto nivel de organización.

${ }^{4}$ Aceptar la idea de que la estabilidad se reduce a la manipulabilidad implica hacer una distinción tajante (y epistemológicamente significativa) entre la física experimental y otras ciencias. Así, Hacking implícitamente asume que es posible hacer una distinción entre la selección natural y el tipo de selección artificial (intencional) que entra 
tradicional se detiene en el nivel de organismos. Los genes son parte de la célula, las células son parte de los órganos, y los órganos son parte de los organismos. Como David Hull y otros han hecho ver, ${ }^{5}$ la presencia de un límite superior en la jerarquía taxonómica y límites análogos en otras jerarquías es una suposición artificialmente restrictiva para nuestra comprensión de lo que es un proceso evolutivo. La jerarquía taxonómica no debe verse basada en la relación de pertenencia a clases, sino basada en una relación de parte a todo. En las jerarquías basadas en la relación de parte a todo, todos los entes son del mismo tipo, todos son individuos. No se asume que exista un nivel básico inferior de organismo (o genes) o cualquier otra unidad preferida de uno de los tantos programas reduccionistas. En este sentido de jerarquía los fenómenos forman una jerarquía evoluti$v a$. En esta jerarquía podemos detectar una direccionalidad en cada línea de desarrollo. Una línea de desarrollo es una serie de fenómenos que pueden ordenarse en términos de la relación de parte a todo de manera única. Si $A$ es un fenómeno en la línea de desarrollo de $B$ entonces la creación de $A$ se requiere previamente para la creación de $B$.

Nótese que el orden de la creación de los fenómenos en la historia de la ciencia no corresponde por lo general al orden de la línea de desarrollo de los fenómenos en la evolución biológica. El mecanismo de la transmisión de los caracteres hereditarios en el nivel cromosómico se constituyó primero como fenómeno fundador, sólo tiempo después se logró

en la creación de un fenómeno en la física. Sin embargo, el hecho de que algunos fenómenos sean creados intencionalmente es algo significativo, pero no es la característica distintiva de la noción de fenómeno que es importante rescatar de las tradiciones experimentales. Es más importante la capacidad del fenómeno para atrincherarse en la creación de otros fenómenos y, con respecto a su atrincheramiento, el hecho de que un fenómeno sea "artificial" desempeña un papel secundario.

5 Véase Hull, 1988; Ghiselin, 1974; Eldredge, 1985. 
encontrar un modelo satisfactorio de los mecanismos subyacentes en el nivel molecular. Las líneas de desarrollo de fenómenos son, por así decir, el esqueleto de los conceptos cambiantes de la ciencia. Los conceptos en tanto que se refieren a jerarquía de fenómenos cambian porque cambian (se refinan o se agregan) los subconceptos constituyentes. Pero no cambian de manera que la direccionalidad de una línea de desarrollo de fenómenos no implique a su vez un aumento del conocimiento.

El sentido en el que un fenómeno entra en la creación de otro fenómeno no es el sentido en el que un volante en particular (hecho por la fábrica Omega el 17 de junio de 1966, por ejemplo) entra en la creación de un mecanismo particular de relojería. Más bien, un fenómeno entra en la creación de otro fenómeno en el sentido en que forma parte de él, como concepto. Así, es claro que este "formar parte" no es algo dado en el mundo independientemente de nuestros conceptos, sino más bien es la manera que tenemos de hacer claro el contenido empírico de aquéllos.

Laudan (en 1977) pensaba que el progreso podía caracterizarse en términos de la capacidad para resolver problemas. Qué es un problema se reduce, para Laudan, a un intento por clasificar los problemas, que si bien resulta útil a veces, globalmente es ad hoc. La clasificación que hace Laudan de los problemas en la ciencia es análoga a la clasificación en la biología pre-darwiniana, es en última instancia arbitraria. Lo que estoy sugiriendo es que una clasificación de los problemas empíricos y conceptuales en la ciencia debe subordinarse a una reconstrucción histórica de las lineas de desarrollo de los fenómenos. Esto constituye un referente objetivo para la noción de progreso.

Esta noción de progreso no es una noción global de progreso, no ordena toda la historia de la ciencia a lo largo de una dirección como la concepción tradicional suponía que debía hacerlo toda noción de progreso. Pero no es 
una noción que sólo sea aplicable en "periodos de ciencia normal" o que, más en general, requiera o presuponga una medida comparativa de contenido empírico. El progreso puede detectarse por medio de una reconstrucción histórica pero no requiere de una medida del contenido empírico.

A diferencia de las propuestas de Lakatos y Laudan, la propuesta presente se basa en una reconstrucción histórica que no admite reconstrucciones significativamente diferentes (con respecto a la reconstrucción de líneas de desarrollo de fenómenos). La estabilidad de los fenómenos, a diferencia de la clasificación y jerarquización de problemas de Laudan, por ejemplo, es una característica objetiva de la historia de la ciencia. El progreso de una línea de fenómenos es objetivo en el mismo sentido en el que Hacking dice que el efecto fotoeléctrico "está aquí para quedarse". Se apela en última instancia a un criterio pragmático de lo real.

La creación de un fenómeno puede hacerse al margen de una teoría, pero siempre implica conceptos. El lanzamiento de dados es un fenómeno azaroso, pero si bien han existido juegos de azar desde mucho tiempo atrás, y tenemos pruebas arqueológicas de la existencia de proto-dados (que posiblemente eran utilizados en rituales religiosos) hace miles de años, el fenómeno del lanzamiento de dados no puede decirse que haya existido hasta que el concepto de una distribución azarosa surgió en el siglo XVII. No es suficiente manipular un dado, el fenómeno azaroso surge a la par del concepto de azar.

9. La relación entre los conceptos de progreso y evolución

Si creemos que hay leyes de la naturaleza "allá afuera" que nosotros descubrimos y vamos organizando como en un rompecabezas, entonces la noción de progreso en la ciencia es simplemente la idea de progreso como acumulación de 
leyes (o de los hechos explicados por aquéllas). Pero si, como he hecho ver, no hay manera de hacer una distinción tajante entre los aspectos pasivos y activos que entran en la creación de un fenómeno, la idealización de lo que es una ley no tiene fundamento. Con este supuesto, la presente propuesta es que podemos caracterizar el progreso sobre la base de una cierta extensión de lo que es un proceso evolutivo. Para precisar mi propuesta es necesario aclarar la relación entre los conceptos de evolución y progreso. Principio con un breve resumen de la larga controversia sobre este tema.

La idea de la gran cadena del ser, el tema del libro clásico de Lovejoy (Lovejoy, 1936), es uno de los grandes temas de las culturas indoeuropeas. La idea de que tanto la materia como todos los organismos están ordenados en una cadena ascendente que va hacia lo más perfecto es indudablemente el núcleo de la idea tradicional de progreso. La idea de progreso fue popularizada por los intelectuales de la ilustración, y en el siglo XIX se cuenta entre sus adherentes prácticamente a todos los científicos y filósofos connotados. Adam Smith, Condorcet, Lamarck, Comte, Hegel y Marx son típicos exponentes de esta doctrina. Spencer es famoso por su detallada presentación y aplicación sistemática de la idea de que el progreso biológico y el progreso en la cultura son el producto de una ley universal que hace que lo simple pase a lo complejo, y lo homogéneo a lo heterogéneo (Spencer, 1857).

El desarrollo de la teoría de la evolución de Darwin fue tomado hasta muy recientemente como el momento histórico que, por lo menos en retrospectiva, permite hacer la distinción entre evolución y progreso. Deberíamos evitar - nos dice Darwin - hablar de la evolución en términos de peor y mejor. ${ }^{6}$

6 Un examen del darwinismo ortodoxo como punto de partida para 
Pero las cosas no son tan simples. El mismo Darwin parece haber asumido cierta idea de progreso cognoscitivo como parte de una tendencia general hacia la perfección de los procesos naturales (véase Richards, 1992). Ruse, en 1988, hace ver que tanto Darwin como la mayoría de los biólogos arquitectos de la síntesis en biología a principios de este siglo siguen sosteniendo, a pesar de su rechazo explícito de una doctrina progresivista, una serie de supuestos íntimamente asociados con el concepto de progreso. ${ }^{7}$

Como Gould nos dice en 1982 (y otros escritos), en contraposición al reduccionismo genético de Dawkins y muchos otros biólogos contemporáneos es necesario pensar en la evolución como un proceso jerárquico. El mundo orgánico está constituido por estadios: genes, células, organismos, poblaciones, especies. Si bien los procesos en un nivel se consideran dependientes de procesos en otro nivel, estos procesos no pueden reducirse a procesos que suceden en un nivel fundamental - como el nivel genético de Dawkins, por ejemplo. Gould considera que esta idea de un proceso evolucionista jerárquico permite explicar la autonomía del dominio de la cultura y de la ciencia en particular como inmersos en un proceso evolutivo, pero sin pretender una reducción a un proceso único. Esta idea está incorporada en mi explicación de la noción de progreso cognoscitivo en términos de la jerarquía de fenómenos-conceptos. Visto desde esta perspectiva, el progreso cognoscitivo consiste en la evolución de conceptos asociada con la construcción de una jerarquía de fenómenos en la ciencia.

Esta concepción generalizada de proceso evolutivo se examina en otra parte (Martínez, 1992a). Mi argumento

la elaboración y crítica de una noción de progreso se encuentra en Castrodeza, 1988.

7 Con respecto a este tema véase también el capítulo V de Castrodeza, 1988. 
aquí no descansa en los detalles de cómo deba entenderse esta generalización de un proceso evolutivo en la historia misma de la ciencia. Más bien, se basa en el hecho de que la distinción tradicional entre procesos adaptativos "naturales" y procesos adaptativos "artificiales" descansa en una distinción entre hechos y leyes que no es sostenible. Si partimos de la idea de que la evolución orgánica, como la historia de la ciencia, implica esencialmente la creación de mecanismos (y de sus niveles de organización asociados) en el marco de la construcción de líneas de fenómenos, entonces debe quedar claro que ambos - la historia de la ciencia y la evolucion orgánica - comparten cierto sentido de progreso. Una idea de progreso que no consiste en la acumulación de leyes (o de los hechos que explican) sino en la creación de mecanismos y en el atrincheramiento de esos mecanismos en fenómenos estables. ${ }^{8}$

Kuhn sugiere, en 1991 (p. 9), que el origen de la estabilidad de los fenómenos es una "cosa en sí” kantiana más allá de la experiencia. Yo estoy sugiriendo una posición más radicalmente evolucionista en historiografía como el núcleo de un proyecto de la naturalización de la epistemología. Kuhn reconoce que la historia no tiene acceso a un plano arquimideano para desde allí hacer una reconstrucción pri-

8 En la terminología de Ayala — en Nitecki, 1988-, la variedad del concepto de progreso que propongo es un tipo de progreso no uniforme y particular. Una noción de progreso es no uniforme ("net progress") si no se requiere que el progreso sea una característica uniforme de un proceso. Es posible que haya fluctuaciones, lo importante es que el resultado final, considerando un intervalo de tiempo suficientemente grande, sea un aumento del parámetro que tomamos como referencia para medir el progreso; en nuestro caso, este parámetro sería la complejidad de los mecanismos y de los niveles de organización asociados con esos mecanismos. Un progreso particular es un tipo de progreso que ocurre en algunas de las secuencias históricas que pueden considerarse pero no en todas (en Ayala, 1988, pp. 78-80). 
vilegiada que pueda identificarse con lo objetivo. De esto no se sigue, sin embargo, que la fuente de la estabilidad esté más allá de la experiencia. Todo lo que se sigue es que la objetividad en la ciencia no es algo independiente de nuestros conceptos.

\section{Conclusión}

Usualmente se trata de caracterizar el progreso mediante un análisis del cambio conceptual en teorías. Implícitamente se trata de establecer una noción de progreso interna en las tradiciones teóricas. La pregunta problemática es entonces ¿en qué sentido podemos decir que nuestras teorías progresan? Esto nos lleva a las dificultades ampliamente debatidas en la filosofía de la ciencia sobre la relación entre racionalidad, progreso y explicación. Introduciendo el concepto de tradición científica, y en particular la noción de tradición experimental como aquel tipo de tradición centrada en la creación y el atrincheramiento de fenómenos, es posible formular y responder al problema de caracterizar el progreso científico de manera diferente.

Una vez que centramos nuestra atención en mecanismos y fenómenos, como el resultado estable e históricamente acumulable de la actividad científica, es posible decir sin más que el progreso de la ciencia consiste en la formación o creación de líneas de desarrollo (de atrincheramiento) de fenómenos. La ciencia progresa en la medida en la que se va creando una compleja red de fenómenos que se van atrincherando en la historia de la ciencia.

La descripción del proceso del atrincheramiento de los fenómenos, como toda historia de la ciencia, requiere de una reconstrucción que se hace con base en conceptos, y es por medio de estos conceptos que podemos aclarar la noción de objetividad que va asociada con el proceso del atrincheramiento de fenómenos. La diferencia con recons- 
trucciones de teorías es que una reconstrucción del proceso de atrincheramiento no busca la reconstrucción verdadera o más aproximada a la verdad. La subdeterminación de la evidencia para la reconstrucción de líneas de fenómenos no nos permite tener la certeza de que todos los fenómenos de una línea de desarrollo se han incorporado en una reconstrucción histórica, así como la subdeterminación de la evidencia puede no permitirnos reconstruir con certeza los orígenes de la vida o los inicios del cosmos o si nos estamos aproximando a la verdad por medio de una teoría en particular; pero esta subdeterminación no es un problema para nuestra concepción de progreso.

En la creación de un fenómeno están incorporados como partes los diferentes fenómenos que son relevantes para esta creación. La subdeterminación de la evidencia es un problema serio si pensamos que hay un punto de partida causal o una visión completa del mundo con respecto a la cual debemos comparar el avance de las teorías. La negación de un nivel básico ontológico-causal suprime de entrada la posibilidad de encontrar una noción de progreso cognoscitivo basada en una medida de la adecuación de las teorías a la realidad.

Si pensamos que el mecanismo de la selección natural actúa sólo en el nivel de genes o de individuos concretos, entonces podemos pensar que el mecanismo de la selección natural es un mecanismo bien delimitado que directamente sólo tiene que ver con el atrincheramiento de un tipo de fenómeno, los diferentes tipos de especies. Pero si pensamos (como piensan muchos biólogos en la actualidad) que el mecanismo de la selección natural actúa simultáneamente pero de manera distinta en los diferentes niveles de la jerarquía evolucionista, entonces la analogía con el proceso de atrincheramiento de fenómenos es más fructífera. Po- 
demos, incluso, pensar que los mecanismos evolutivos que llevan a la creación de especies son parte de una jerarquía que actúa de maneradiferente (pero relacionada) en distintos contextos, y que el proceso de atrincheramiento de fenómenos en la historia de la ciencia es el producto de mecanismos similares a los que generan los fenómenos biológicos. ${ }^{9}$

La noción de progreso sugerida arriba no implica la idea de aproximación a la verdad. Presupone que la objetividad de la ciencia se articula en el proceso histórico del atrincheramiento de fenómenos. Presupone también que detrás de la noción de progreso hay una noción de racionalidad práctica que se manifiesta en la construcción de líneas de desarrollo de fenómenos. Estos presupuestos son característicos de las tradiciones experimentales en la ciencia

En lugar de tratar de encontrar en la biología un análogo de la noción de progreso del siglo XIX (como se ha tratado de hacer innumerables veces) debemos tratar de extender a toda la ciencia una noción importante de progreso que está implícita en la biología. El conocimiento científico progresa en la medida en que las líneas de desarrollo de fenómenos siguen ramificándose en nuevos fenómenos y conceptos que nos permiten tener una idea más correcta de los mecanismos de creación (y de estabilización) de fenómenos en el mundo y en la historia de la ciencia.

9 Hull en 1988 recalca la importancia que tiene la búsqueda de los mecanismos concretos de la evolución en el contexto sociológico de la ciencia. Me parece, sin embargo, que la noción de mecanismo de Hull es demasiado estrecha. En la terminología de Fleck que he adaptado a mis propósitos, yo diría que el problema es que el supuesto mecanismo de Hull (la búsqueda de crédito y reconocimiento por parte de los científicos) a lo sumo describe los aspectos "activos" (sociológicos) de un proceso, y deja de lado la búsqueda de los aspectos "pasivos" que son parte esencial de la caracterización de cualquier mecanismo. 


\section{BIBLIOGRAFÍA}

Almond, G., M. Chodorow y R. Pearce, 1982, Progress and its Discontents, University of California Press, Berkeley.

Ayala, F., "Can 'Progress' be Defined as a Biological Concept?", en Nitecki, 1988, pp. 75-96.

Bendall, D. (comp.), From Molecules to Men, Cambridge University Press, Cambridge.

Bechtel, W., 1990, "Scientific Evidence: Creating and Evaluating Experimental Instruments and Research Techniques", en Arthur Fine, Micky Forbes y Linda Wessels (comps.), PSA 1990 (Proceedings of the 1990 Biennial Meeting of the Philosophy of Science Association), Philosophy of Science Association, East Lansing, Michigan, vol. I.

Bernard, C., 1865, Introduction a l'Étude de la Médicine Experimentale, Collège de France, París.

—_, 1989, Antología, en José Luis Barona (comp.), Ediciones Península, Barcelona.

Bohr, N., 1934, "The Quantum Postulate and the Recent Development of Atomic Theory", en Atomic Theory and the Description of Nature, Cambridge University Press, Cambridge. Trad. castellana en Alianza Editorial.

— 1935, "Quantum Mechanics and Physical Reality", $N a$ ture, no. 136. Una versión revisada y aumentada fue publicada en Physical Review, no. 48, 1935, pp. 696-702.

—, 1949, "Discussion with Einstein on Epistemological Problems in Atomic Physics", en P.A. Schilpp (comp.), Albert Einstein: Philosopher Scientist, Library of Living Philosophers, Evanston (Ill.).

Castrodeza, Carlos, 1988, Ortodoxia darwiniana y progreso biológico, Alianza Universidad, Madrid.

Carlson, Elof A., The Gene, a Critical History, en Saunders, 1966. Reimpreso en 1989 en History of Science and Technology Reprint Series, Iowa State University Press.

Cartwright, Nancy, 1983, How the Laws of Physics Lie, Oxford University Press, Oxford.

Darden, Lindley, 1989, Theory Change in Science, Oxford University Press, Oxford. 
Dawkins, R., 1986, The Blind Watchmaker, Longmans, Londres.

De Fontenelle, B., 1982, Conversaciones sobre la pluraridad de los mundos, edición preparada por A. Beltrán Mari, Editora Nacional, Madrid.

Eldredge, Niles, 1985, Unfinished Synthesis, Biological Hierarchies and Modern Evolutionary Thought, Oxford University Press, Nueva York/Oxford.

Fleck, L., 1935, Die Entstehung und Entwicklung einer Wissenschaftlichen Tatsache, Suhrkamp Verlag, 1980. Textualmente idéntica a la edición de 1935 publicada por Schabe \& Co. Trad. castellana en Alianza Editorial.

Gallison, P., 1987, How Experiments End, The University of Chicago Press, Chicago.

Ghiselin, M., 1974, "A Radical Solution to the Species Problem", Systematic Zoology, no. 215, pp. 207-215.

Gould, S.J., 1982, "Darwinism and the Expansion of Evolutionary Theory", Science, no. 216, pp. 380-387.

Hacking, I., 1983, Representing and Intervening, Cambridge University Press, Cambridge. Trad. castellana de S. Martínez (en prensa).

Hull, D., 1988, Science as a Process, The University of Chicago Press, Chicago.

Kuhn, T., 1970, The Structure of Scientific Revolutions, segunda edición aumentada. En la enciclopedia Foundations of the Unity of Science, University of Chicago Press, Chicago, vol. II, no. 2.

_, 1977, "Mathematical versus Experimental Traditions in the Development of Physical Science", en The Essential Tension, The University of Chicago Press, Chicago.

— , 1991, "Presidential Address: The Road since Structure", PSA 1990 (Actas del Congreso de 1990), Philosophy of Science Assoc., vol. II.

Lakatos, I., 1970, "Falsification and the Methodology of Scientific Research Programmes", en A. Musgrave y Lakatos (comps.), Criticism and Growth of Knowledge, Cambridge University Press.

Laudan, L., 1973, "Peirce and the Trivialization of the SelfCorrecting Thesis", en R. Giere y R. Westfall (comps.), 
Foundations of Scientific Method: The Nineteenth Century, Indiana University Press, Bloomington.

_, 1977 , Progress and its Problems, University of California Press, Berkeley.

__, 1990, Science and Relativism. Some Key Controversies in the Philosophy of Science, The University of Chicago Press, Chicago.

Lovejoy, A., 1936, The Great Chain of Being, Harvard University Press, Cambridge.

Martínez, S., 1990, "Más allá de la presuposición newtoniana", Crítica, no. 66.

—_, 1991, “¿Qué es una ley irreductiblemente estadística?”, Diánoia, 1991.

— $1992 \mathrm{a}$, "The Roots and the Epistemological Implications of the Autonomy of Scientific traditions" (en preparación).

—_, 1992b, "Una respuesta al desafío de Campbell: la evolución y el atrincheramiento de las reglas heurísticas", en S. Martínez y León Olivé (comps.), Evolución y conocimiento (en prensa).

- 1992c, "Objetividad contextual y robustez", Diánoia, 1992.

Mill, J.S., 1874, A System of Logic, 8a. ed., Nueva York.

Nitecki, M., 1988, Evolutionary Progress, The University of Chicago Press, Chicago/Londres.

Oakley, F., 1961, "Christian Theology and the Newtonian Science: The Rise of the Concepts of the Laws of Nature", Church History, no. 30, pp. 433-457.

Pickering, A., Constructing Quarks, The University of Chicago Press/The University of Edinburgh Press, Chicago/Edimburgo.

Popkin, Richard, 1979, The History of Scepticism from Erasmus to Spinoza, University of California Press, Berkeley.

Ruby, Jane E., 1986, "The Origins of Scientific Law", Journal of the History of Ideas, no. 47, pp. 341-359.

Richards, Robert, 1992, The Meaning of Evolution, The University of Chicago Press, Chicago.

Ruse, M., "Molecules to Men: Evolutionary Biology and Thoughts of Progress", en Nitecki, 1988, pp. 97-128.

Spencer, H., 1857, "Progress: Its Law and Cause", Westminster Review, reimpreso en Essays: Scientific, Political and Speculative, 1868. 
Toulmin, S., Human Understanding, Princeton University Press, Princeton.

Van Fraassen, Bas C., 1989, Laws and Symmetry, Clarendon Press, Oxford.

, 1991, Quantum Mechanics, Clarendon Press, Oxford.

Recibido: 11 de enero de 1993 


\section{SUMMARY}

In this paper I address the problem of characterizing a notion of scientific or cognitive progress. I show that there is a close connection between the different characterizations of scientific method and notions of progress. Until the early sixties, a very common (often implicit) assumption was that progress could be understood as a by-product of the use of the scientific method. The work of Thomas Kuhn made clear that such a simple minded characterization of progress could not be right. I examine a series of attempts aiming to spell out a notion of scientific progress, and argue that an underlying assumption of these proposals is an assumption about the underlying methodological unity of science.

I claim that it is important for the correct formulations of a series of problems in the philosophy of science, and for the problem of characterizing progress in particular, to draw a distinction among different sorts of traditions in science. Each tradition articulates different explanatory aims, methods and notions of progress. In this paper I spell out a notion of cognitive progress that is implicit in experimental traditions. In future work I will discuss the notions of progress associated with other traditions.

In experimental traditions progress is understood in terms of the stabilization of phenomena and their entrenchment. A phenomenon gets entrenched when it is used for the construction of other phenomena. In order to understand the epistemological significance of the entrenchment of phenomena it is necessary to show that the traditional understanding of laws of nature in theoretical traditions, and in most philosophical accounts, is not suitable to understand the sense in which experimentalists talk of the "manipulation of laws of nature". I provide a series of examples in order to clarify to concepts of phenomenon and entrenchment of phenomena which are important in experimental traditions. I suggest that the process of entrenchment of phenomena can be understood as a generalized evolutionary process. In that way, the analogy between progress and evolution, a topic of heated discussions for over a century, can help to elucidate the dynamics of experimental traditions in science. 